\title{
Analysis of sequenced chromosome-specific libraries of gekkonids sheds light to large scale genome reshuffling in reptiles
}

\author{
Katerina Tishakova \\ Institute of Molecular and \\ Cellular Biology SB RAS \\ Novosibirsk, Russia \\ Novosibirsk State University \\ Novosibirsk, Russia \\ k.tishakova@g.nsc.ru \\ Dmitry Prokopov \\ Institute of Molecular and \\ Cellular Biology SB RAS \\ Novosibirsk, Russia \\ dprokopov@mcb.nsc.ru
}

\author{
Ilya Kichigin \\ Institute of Molecular and \\ Cellular Biology SB RAS \\ Novosibirsk, Russia \\ kig@mcb.nsc.ru \\ Anna Molodtseva \\ Institute of Molecular and \\ Cellular Biology SB RAS \\ Novosibirsk, Russia \\ rada@mcb.nsc.ru \\ Lukáš Kratochvil \\ Charles University \\ Prague, Czech Republic, \\ lukkrat@email.cz
}

\author{
Artem Lisachov \\ Institute of Cytology and \\ Genetics SB RAS \\ lisachov@bionet.nsc.ru \\ Vladimir Trifonov \\ Institute of Molecular and \\ Cellular Biology SB RAS \\ Novosibirsk, Russia \\ Novosibirsk State University \\ Novosibirsk, Russia \\ vlad@mcb.nsc.ru
}

\begin{abstract}
Representatives of Infraorder Gekkota demonstrate relatively low chromosomal evolution and rapidly evolved sex chromosome systems. Using low-coverage chromosome sequencing, we sequenced chromosome-specific libraries of Gekko japonicus and putative sex chromosome of Hemidactylus platyurus to compare with annotated genomes of squamate reptiles. We found evolutionary long conservation of macro-chromosome and high dynamic of micro-chromosomes. Also, we discovered homologues synteny blocks between sex chromosomes of species from different infraorder.
\end{abstract}

Keywords - Karyotypic evolution, macro- and microchromosomes, sex chromosomes, Gekko japonicus

Introduction

Infraorder Gekkota represents a specious infraorder of reptiles, taking a basal position relative to a large clade of squamate reptiles, including snakes, iguanids, scincids, lacertids and anguimorphs. The infraorder is characterized by unimodal karyotype without macro- and micro-chromosomes. The ancestral karyotype is known to have consisted of 38 acrocentric chromosomes, and modern karyotypes differ from it by a relatively small number of chromosomal rearrangements [1]. Also, geckos demonstrate the rich diversity of sex chromosome systems [2]. However, most chromosome-wide assemblies of the genome are available for lacertids, snakes, varanids and iguanids are still missing in Gekkota. Here we applied low-coverage chromosome sequencing to evaluate the genetic content in a representative of geckos - Gekko japonicus and putative sex chromosome of Hemidactilus platyrus to compare with annotated genomes of squamate reptiles and study homologous synteny blocks and evolutionary breakpoints regions.

\section{Metods}

The chromosome-specific libraries of Gekko japonicus were prepared from chromosome sorted with a dual laser cell sorter (Mo-Flo, Dako) at the Cambridge Resource Center for Comparative Genomics, Department of Veterinary Medicine, University of Cambridge, Cambridge, UK, as described previously [3]. Libraries were amplified and labeled using DOP-PCR, chromosome specificity of libraries was verified by FISH. To prepare chromosome-specific libraries for sequencing, we used TruSeq Nano DNA Low Throughput Library Prep Kit (Illumina). 300 bp paired-end reads were generated on Illumina MiSeq using the Illumina MiSeq Reagent Kit v3 according to the manufacturer's instructions. Sequencing data were processed using the DOPseq_analyzer pipeline [4]. At first, the cutadapt removes of Illumina adapter sequences and DOP primers. The purified reads are aligned on the Lacerta agilis and Crotalus viridis viridis genomes to search for target regions and the human genome to remove contamination reads using BWA, low-quality alignments were discarded. Then, by calculation, the density of alignment, the identification of target regions occurs using DNAcopy package.

\section{Results}

We compared the obtained sequencing data with the annotated genome of Lacerta agilis (Lacertidae, Squamata) and Crotalus viridis viridis (Viperidae, Squamata) and found evolutionary long conservation of macro-chromosome along with high dynamics of micro-chromosomes. We refined the breakpoints in reptile genomes and demonstrated the breakpoint re-use. Also, we found homologues of the putative sex chromosome of Hemidactilus platyurus in the G. japonicus karyotype, which is not a sex chromosome. However, the putative sex chromosome of $H$. platyurus has a synteny block with the sex chromosome of the Noropus sagrey (Iguania, Squamata) and contains the RSPO1 gene. This result suggested that in different reptile lines, the same genetic material can independently be involved in the formation of sex chromosomes.

$$
\text { Acknowledgment }
$$

This work was supported by RFBR 19-54-26017.

\section{References}

[1] Pokorná M.J., Trifonov V., Rens W., Ferguson-Smith M. ., \& Kratochvíl L. (2015). Chromosome Research, 23(2): 299-309.

[2] Gamble T. (2010). Sexual Development, 4(1-2), 88-103.

[3] Yang F., Carter N., Shi L., \& Ferguson-Smith M. (1995). Chromosoma, 103(9): 642-652.

[4] Makunin A.I., Kichigin I.G., Larkin D.M., O'Brien P.C., FergusonSmith M.A., Yang F., ... \& Graphodatsky A.S. (2016). BMC Genomics, 17(1): 618 . 